\title{
Quantitative Amplitude and Phase Imaging with Interferometric Plasmonic Microscopy
}

Yuting Yang, Chunhui Zhai, Qiang Zeng, Ab Lateef Khan, Hui Yu*

Institute for Personalized Medicine, School of Biomedical Engineering, Shanghai Jiao

Tong University, Shanghai, 200030, China

Correspondence should be sent to:

Prof. Hui Yu

Email: hui.yu@sjtu.edu.cn

$\underline{\text { Tel/Fax: }+86-21-62933948}$ 


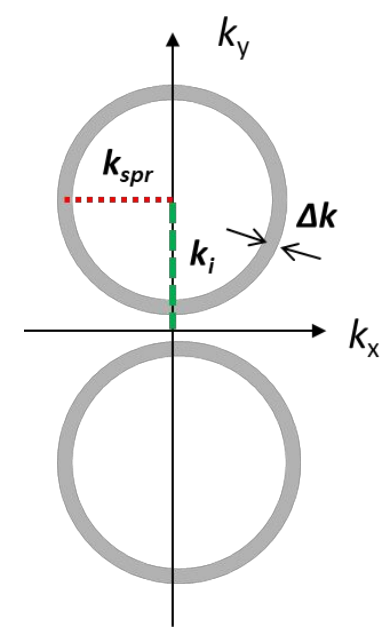

Figure S1 Illustration of wavevector estimation in iPM angular spectrum.

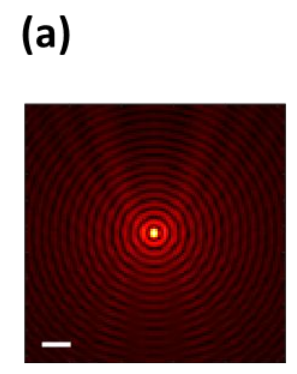

(b)

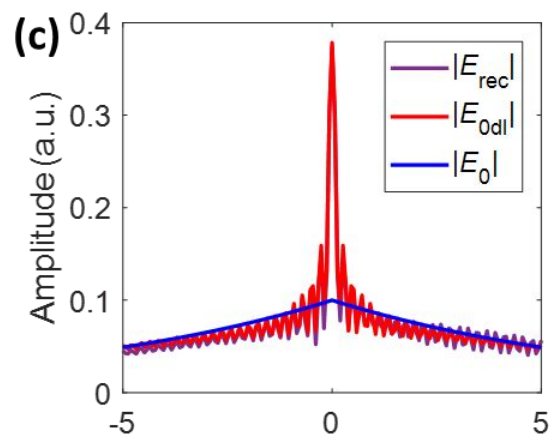

(d)
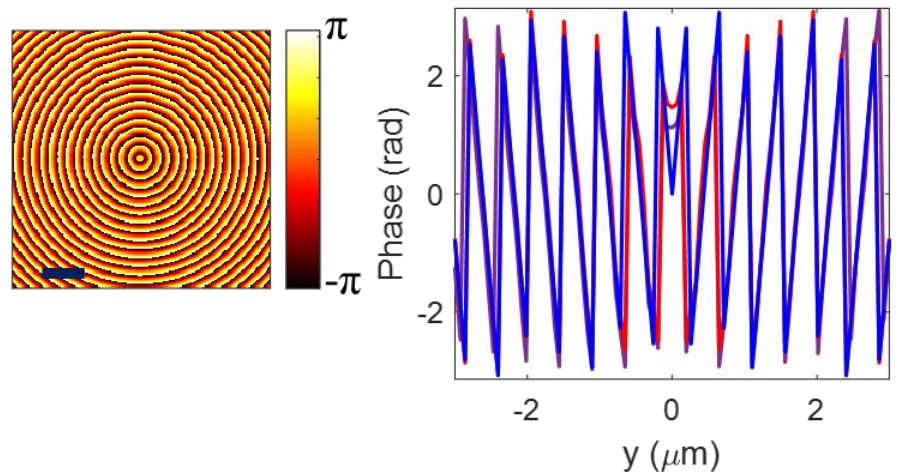

FigureS2 Simulation results of single nanoparticle image. (A) (C) Amplitude and (B)

(D) phase images and profiles of nanoparticle images without (blue) and with (red) pupil function, and reconstructed (purple). 


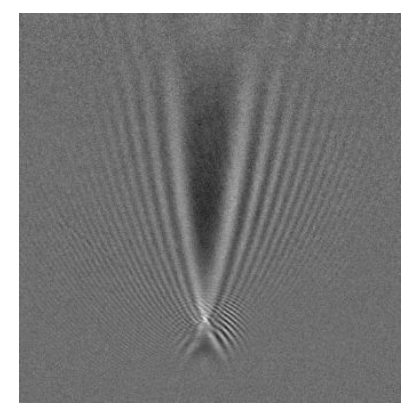

Figure S3 iPM image recorded at $\mathrm{z}=2 \mu \mathrm{m}$ for the reconstruction of $\mathrm{z}$-stack images in Figure 2C.

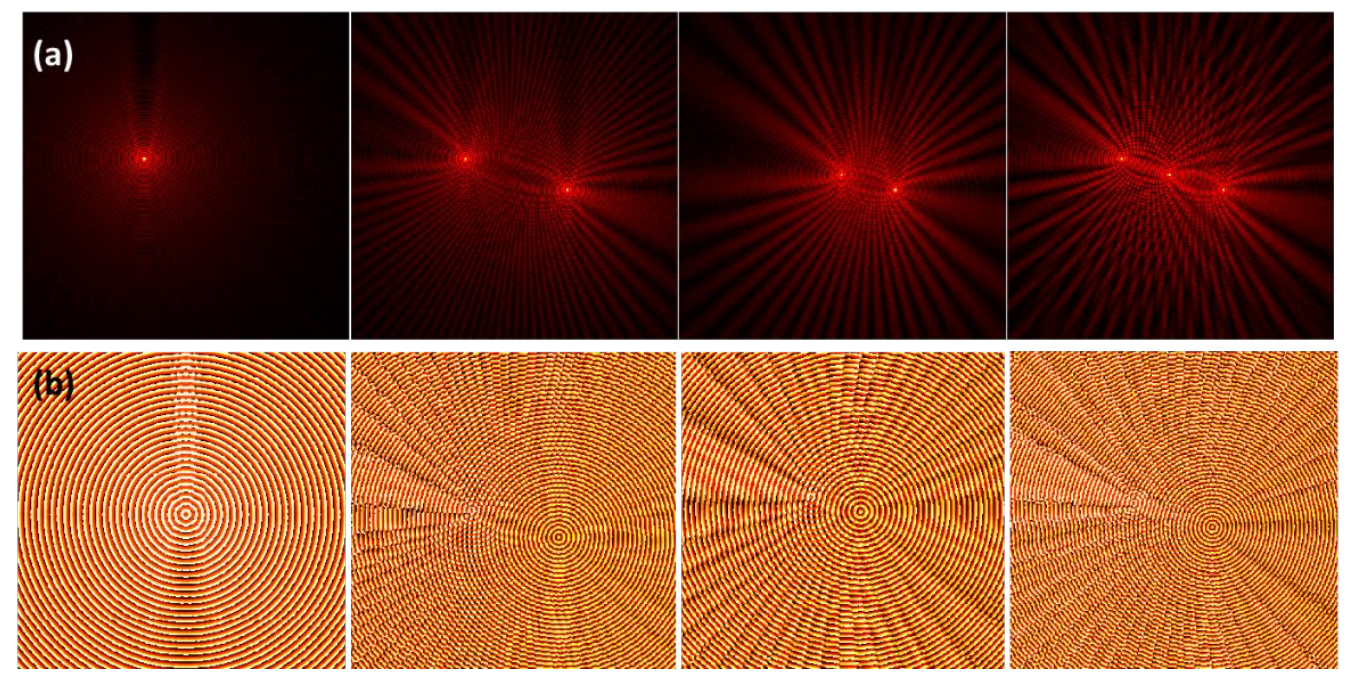

Figure S4 Simulated amplitude (A) and phase (B) images of SP fields scattered by particles.
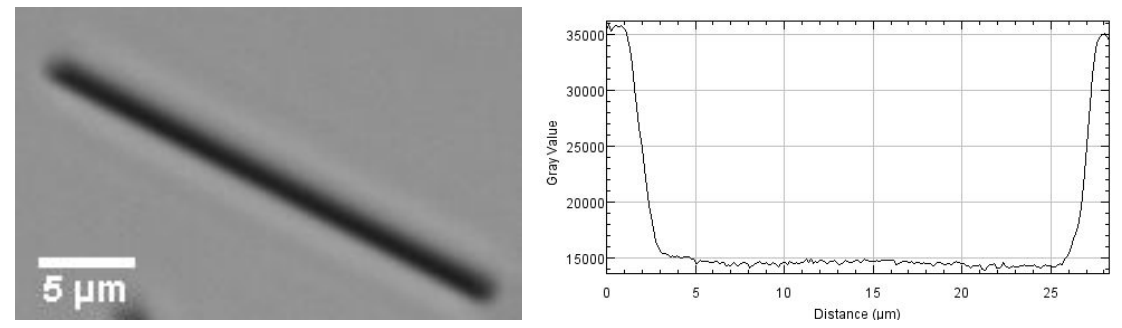

Figure S5 Bright field image of Au nanowires (left) and length profiles. 

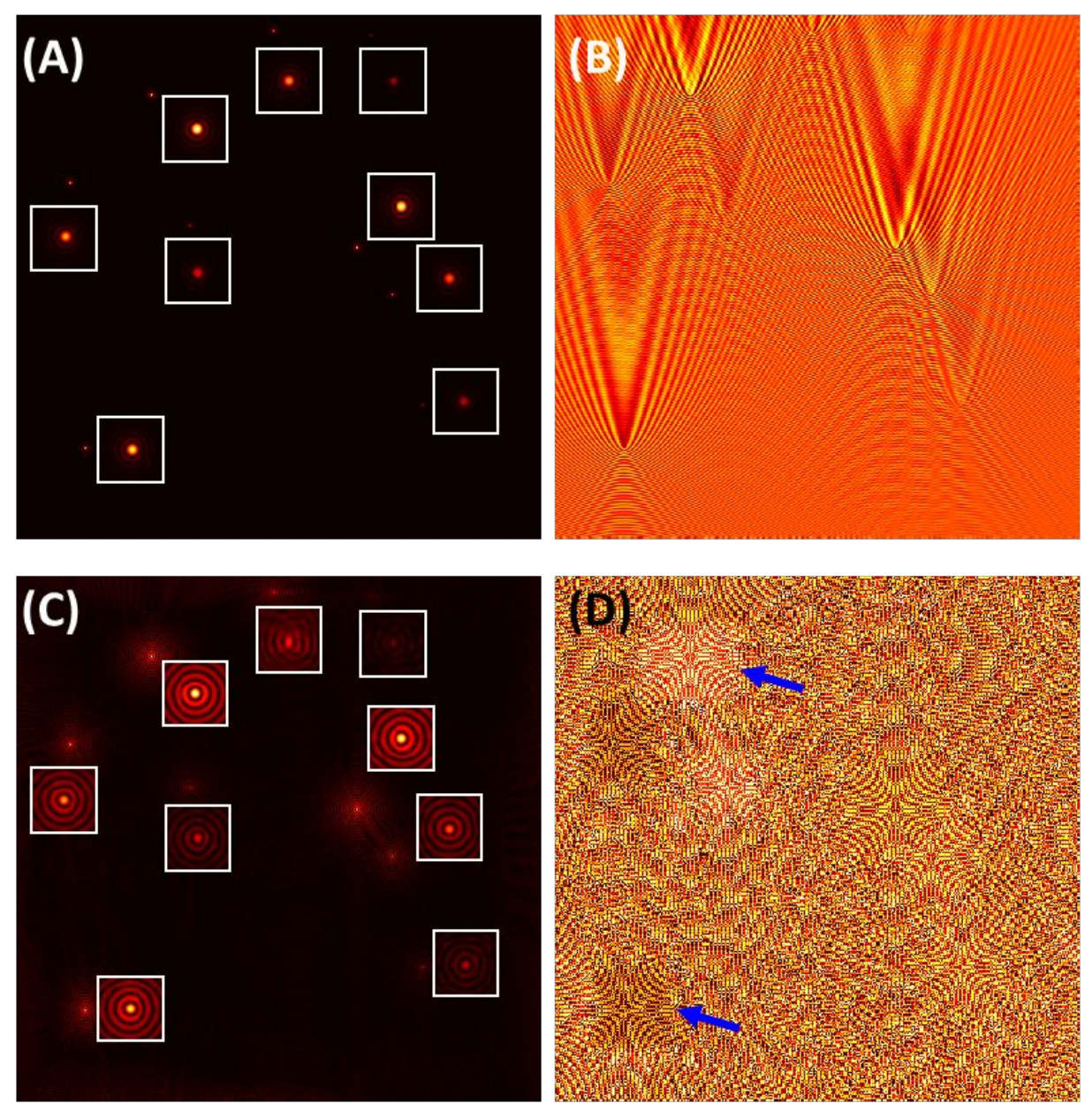

Figure S6 Simulated iPM images of 10 scatterers. (A) Diffraction limited image, (B) iPM image, (C) Reconstructed amplitude image, (D) reconstructed phase image. 